\section{FACTORES INFLUYENTES EN EL BAJO RENDIMIENTO ESCOLAR DESDE UNA MIRADA SOCIAL}

\section{RESUMEN}

El artículo presenta los diferentes factores que influyen en el bajo rendimiento escolar como lo son las situaciones psicológicas y/o sociales en las que se ve envuelto el NNA (niños, niñas y adolescentes). Centrando principal atención en el factor familia, y la determinación que tiene este sobre el rendimiento escolar del estudiante. La investigación se desarrolló por medio de la creación de una valoración socio-familiar, la cual es fundamental en la intervención de Trabajo social. El diseño, la implementación y el análisis de esta valoración se dividió por una serie de instrumentos, los cuales permitieron realizar una debida caracterización e interpretación de la relación del estudiante con el entorno familiar, institucional y como este se desenvuelve individualmente.

Palabras clave: Familiar, Bajo rendimiento escolar, institución educativa, capital cultural, NNA.

\section{INFLUENCING FACTORS ON LOW SCHOOL PERFORMANCE FROM A SO- CIAL VIEW}

\section{Abstract}

The article presents the different factors that influence low school performance, such as the psychological and / or social situations in which the child is involved (children and adolescents). Focusing main attention on the family factor, and the determination that this has on the student's school performance. The research was developed through the creation of a socio-family assessment, which is essential in the intervention of Social Work. The design, implementation and analysis of this assessment was divided into a series of instruments, which allowed for a proper characterization and interpretation of the student's relationship with the family, institutional environment and how it unfolds individually.

Keywords: Family, low school performance, educational institution, cultural capital, NNA.

\title{
Maira Lizeth Cristancho Hoyos
}

Trabajo Social. Estudiante. Código ORCID.0000-0002-5460-2133. Correo electrónico: mcdiecinueve@gmail.com

\section{Luisa Fernanda Fonseca}

Trabajo Social. Estudiante. Código ORCID.0000-0002-9023-1851. Correo electrónico: luisa310398@hotmail.com

* Trabajo de Grado para optar al título de Especialista en derecho de familia.

Corporación Universitaria Republicana.

Recibido: 03-01-2019. Aprobado: 05-09-2019

DOI: http://dx.doi.org/10.21017/pen.repub.2020.n12.a65 


\section{Factores influyentes en el bajo rendimiento escolar desde una mirada social}

\section{Introducción}

En el presente artículo se identificaron los diversos factores que generan el Bajo rendimiento académico en los NNA de grado 6,7 y 8 , del instituto educativo Francisco Miranda, dentro de esta investigación cuantitativa se identificaron que los factores más comunes son: Las situaciones psicológicas, sociales en las que se ve envuelto NNA y la familia.

En la presente investigación se hizo énfasis en la familia, esto se debe a que la familia es el núcleo de fundamental de la sociedad, es el primer sistema con el que una persona crea un primero vinculo, interactúa y tiene un contacto, a partir de esto aprenden a forja una personalidad y conductas que caracteriza a cada individuo. Como lo afirma el autor Muñoz (2005) "La familia es uno de los principales contextos de desarrollo del individuo, por lo que se convierte en el más propicio para la crianza y educación del ser humano, ya que es donde se promueve su desarrollo personal, social y cognitivo" (p. 11)

Esto con el fin de conocer las implicaciones causales que tiene el manejo de las autopercepciones de los estudiantes y de la familia, con respecto al bajo rendimiento académico.

La muestra fue de 35 estudiantes de grados 6,7 y 8 pertenecientes al I.E.D. Francisco de Miranda ubicado en la localidad de Kennedy. La variable de contexto escolar tuvo una influencia directa en el factor familiar y este, a su vez, en el desarrollo positivo del joven, el cual afectó directamente al promedio de los alumnos, el cual permitió plantear cuestionamientos y posibles soluciones desde el área de Trabajo Social para contrarrestar en próximos años el fenómeno del bajo rendimiento escolar. 


\section{Estado del arte del más antiguo al más reciente}

Según el estudio realizado por Alfonso, Rioboo, Blanco, Morán y Enríquez (2007) que tenía como objetivo conocer los determinantes de rendimiento académico derivados de las relaciones familiares. Con una muestra de 3.942 alumnos, representativa de la Comunidad Autónoma de Galicia, extraída de 35 centros escolares públicos y privados, en contextos socioeconómicos y culturales de tipo medio.

Esto significa que el rendimiento académico es más bajo en la misma medida en que son más altos aspectos como la existencia de recompensas/elogios por parte de la familia hacia el sujeto, promesas de regalos o recompensas materiales, haciendo referencia tanto al esfuerzo realizado como a las calificaciones conseguidas. Parece que las motivaciones, incentivos y refuerzos de tipo extrínseco son contrarios al buen rendimiento académico, cuestión y tema que coincide con otras investigaciones realizadas en semejantes condiciones y contextos.

Para concluir las variables familiares que mejor predicen el rendimiento académico del alumnado de ESO y Bachillerato son la Valoración familiar del estudio y la Colaboración familiar con el centro escolar. Ambas variables se consideran muy importantes ya que son las que ejercen una mayor influencia sobre el rendimiento académico del alumnado. ¿Se unen, sin embargo, a estas variables otras importantes como la Valoración de la capacidad y el esfuerzo y Refuerzo familiar del rendimiento, aunque manteniendo ambas variables una correlación negativa el coeficiente? es negativo, con el rendimiento académico de la muestra total del alumnado de educación secundaria (ESO) y, en este caso, de la muestra del alumnado de ESO y Bachillerato. Estos datos coinciden con los encontrados por González Pineda cuando afirmaba que contrariamente a lo que se suele pensar las recompensas y refuerzos externos y contingentes a los logros de los hijos que dispensan los padres no favorecen el rendimiento académico. Se constata que cuanto más realizan este tipo de reforzamiento los padres, más perjudican el auto-concepto académico de los hijos, disminuye la responsabilidad de los hijos frente a los logros y el desarrollo de las aptitudes académicas, y paradójicamente, también el rendimiento académico es más bajo (González Pienda,2003, p.255).

Lo mismo podemos decir de las variables uso de criterios comparativos y ayuda de la familia al trabajo escolar: ambas aportan poco a la ecuación de regresión respectivamente, pero lo hacen, al igual que en el caso anterior, manteniendo una relación negativa con el grupo de alumnos de rendimiento medio global del total de la muestra; es decir, mantienen un poder predictivo mínimo, pero, siempre, en una relación negativa con el rendimiento académico. 
Se comprueba que la variable que posee un auténtico poder predictivo es la Satisfacción familiar con los resultados. Esta variable, que ya ha aparecido reiteradamente en los anteriores análisis tanto de alumnado de la ESO del año 1999, como de la ESO y Bachillerato de los años 2002 y 2005, respectivamente, vemos que se consolida como una variable determinante y significativa del rendimiento académico.

En conclusión, se destaca que la satisfacción y el agrado que el alumnado percibe en su familia cuando obtiene buenos resultados académicos actúan, sin duda, a través de los componentes motivacionales que rigen y orientan dicha conducta de rendimiento. Se une también a esta ecuación de regresión la variable Control familiar y ayudas familiares al estudio con una pequeña proporción de varianza.

En otro tiempo Jiménez (2011), realizó un estudio con el fin de identificar posibles estrategias pedagógicas y didácticas adecuadas para contribuir al mejoramiento del rendimiento académico en estudiantes de básica primaria de la Escuela Rural Mixta la Laguna Dinde, Municipio de Cajibio Cauca.

Se tomo como muestra 20 estudiantes que presentaban bajo rendimiento y posible deserción escolar, a estos se les hizo seguimiento durante 3 periodos consecutivos. en el transcurso de los periodos se aplicaron instrumentos para realizar la debida Caracterización socio-económica y cultural de las familias, esto permitió identificar que los estudiantes con bajo rendimiento escolar pertenecen a hogares disfuncionales, así como la situación económica y la falta de empleo en la región son factores influyentes en este fenómeno.

Por otro lado Guerrero, Cardona y Cuevas (2013), realizaron una investigación que tenía como objetivo determinar los factores de riesgo que se asocian con el bajo rendimiento académico, en niños escolares de dos Instituciones Educativas Distritales (IED) de Bogotá.

Se identificaron cuatro factores que determinan el riesgo del bajo rendimiento académico de un niño en edad escolar: presentar historia de ausentismo o problemas disciplinarios; presentar estado de privación socio afectiva y problemas de maltrato, pertenecer a un hogar con tres o más niños menores de cinco años o ser un niño frecuentemente enfermo.

Además Ferrel, Robert, Mendoza Y Ballestas (2014) el presente estudio de investigación tuvo como objetivo describir los niveles de depresión y autoestima en estudiantes de secundaria con bajo rendimiento académico, de cinco Instituciones Educativas Distritales (IED) de Santa Marta, La investigación fue descriptiva y diseño transversal, con una población de 629 estudiantes y muestra intencional de 140 estudiantes del Grado $10^{\circ}$, clasificados como de 
bajo rendimiento académico, 51\% varones y $49 \%$ mujeres, con edades de 14 a 19 años, Media de 16 años.

Define el fracaso académico, como un problema que afrontan los estudiantes que presentan un rendimiento académico deficiente, generando su deserción o expulsión del sistema educativo, el cual como todo fenómeno complejo se considera de carácter multicausal, entre los cuales los factores psicológicos son importantes. Cabe precisar, que el rendimiento académico se refiere al nivel de logro que puede alcanzar un estudiante en una o varias asignaturas; cuando el estudiante no alcanza este nivel o no muestra a través de sus resultados el dominio en ciertas tareas, se puede decir entonces que presenta bajo rendimiento académico.

El rendimiento académico es también la resultante del complejo mundo que envuelve al estudiante, determinado por una serie de aspectos cotidianos como el esfuerzo, la capacidad de trabajo, la intensidad de estudio, sus competencias, aptitudes, personalidad, atención, motivación, memoria, medio relacional, recursos tecnológicos, entre otros, que afectan directamente el desempeño académico de los individuos. Otros factores adicionales que influyen pueden ser psicológicos o emocionales como la ansiedad, depresión o autoestima, manifestados como nerviosismo, falta o exceso de sueño, incapacidad para concentrarse, apatía y, en casos extremos, depresión profunda y la afectación de otros factores no cognitivos como acontecimientos vitales adversos o problemáticos, como la pérdida de familiares.

Desde el punto de vista de Ochoa la comunicación en la familia y en la escuela podría ser la solución al bajo rendimiento escolar. Se debe tener presente que la educación de los hijos e hijas no es responsabilidad únicamente de la escuela o de la familia. Las dos Instituciones son el complemento para lograr un alto rendimiento escolar y es aquí donde el/la Trabajador/a Social tiene una hermosa tarea. También he podido analizar que existen varios problemas que perjudican de una u otra manera el rendimiento escolar del/la niño/a. La escuela, la familia y la sociedad, en donde los niños y niñas se desarrollan como sujetos de conocimientos, son los contextos que pueden ayudar o perjudicar el rendimiento escolar. Además con las acciones realizadas en este caso, el comportamiento de la niña cambió, mejoró sus calificaciones, los padres en los deberes, acuden periódicamente a la escuela para conocer el rendimiento escolar de su hija, asisten a terapia familiar y reciben orientación respecto a sus obligaciones en la educación de la niña (2015).

De la misma manera Barrios y Frías (2016) que tenía como objetivo analizar las variables que están influyendo en el aprovechamiento escolar de los jóvenes y basados en una muestra de 250 estudiantes regulares de nivel medio superior. Se estimaron estadísticas uní variadas, donde se calcularon índices y se probó 
un modelo de ecuaciones estructurales en el que se formaron 2 factores: desarrollo positivo del joven y recurso institucional familiar.

El aporte fundamental de este trabajo fue especificar los efectos de los recursos familiares y escolares en el desarrollo positivo del adolescente y su resultado en el rendimiento escolar. En el modelo estructural, el desarrollo positivo del joven tuvo un efecto directo en el promedio escolar lo cual significa que cuanto más auto-determinado, auto-eficaz, auto-regulado y espiritual sea el joven, cuanto más se involucre pro-socialmente con sus compañeros y maestros, y posea una identidad positiva y una habilidad cognitiva, mayor será el promedio que obtenga en la evaluación de un determinado periodo escolar.

Por otra parte, el modelo también muestra cómo el recurso institucional familiar tiene un efecto en el desarrollo positivo del joven. Esto implica que entre mejor sea la relación con los padres, la comunicación con la madre, y el apoyo que tengan los jóvenes de sus padres ya sea emocional o instrumental, mayor será el desarrollo positivo del joven.

\section{Marco Teórico}

El estudio del rendimiento académico de los estudiantes es, por su relevancia y complejidad, uno de los temas de mayor controversia en la investigación educativa, y se le ha dedicado especial atención en las últimas décadas, se tratará de presentar una aproximación conceptual al constructo del rendimiento escolar, contextualizándolo con la realidad que acontece en las aulas de la educación básica regular. El rendimiento escolar es una problemática que preocupa hondamente a estudiantes, padres, profesores y autoridades; y no solo en nuestro país, sino también en otros muchos países latinoamericanos y de otros continentes.

El rendimiento escolar según Cortés (s.f.) lo define como:

"Nivel de conocimiento de un alumno medido en una prueba de evaluación. En el rendimiento académico, intervienen además del nivel intelectual, variables de personalidad (extroversión, introversión, ansiedad...) y motivacionales, cuya relación con el rendimiento académico no siempre es lineal, sino que está modulada por factores como nivel de escolaridad, sexo, actitud."

Por su parte Retana (s.f.) lo define como "El nivel de conocimiento expresado en una nota numérica que obtiene un alumno como resultado de una evaluación que mide el producto del proceso enseñanza aprendizaje en el que 
participa" Partiendo de estos dos breves conceptos se tiene como referencia que el rendimiento escolar es un nivel medido por calificaciones numéricas, que obtienen los alumnos de acuerdo a una serie de variables y dentro de este se ven inmersos distintos factores que van a intervenir.

Finalmente se mencionará a Ruiz (2002, p. 52) dice al respecto: "El rendimiento escolar es un fenómeno vigente, porque es el parámetro por el cual se puede determinar la calidad y la cantidad de los aprendizajes de los alumnos y, además, porque es de carácter social, ya que no abarca solamente a los alumnos, sino a toda la situación docente y a su contexto”.

Como el anterior autor lo define el rendimiento escolar es de carácter social pues no afecta solo a los estudiantes sino a los sistemas en los que este se encuentra inmerso (familia, contexto institucional). El bajo rendimiento escolar o académico, es el resultado de múltiples factores y causas, entre los que se encuentran: los de carácter individual con relación a su estado de salud, el contexto familiar, la situación socioeconómica y otros asociados al propio sistema educativo. (Guerrero, Cardona, Cuevas, 2013, p.656).

Diversos autores coinciden al sostener que el rendimiento académico es el resultado del aprendizaje suscitado por la actividad didáctica del profesor y producido en el alumno. Para Martínez-Otero (2007), desde un enfoque humanista, el rendimiento académico es "el producto que da el alumnado en los centros de enseñanza y que habitualmente se expresa a través de las calificaciones escolares" (Citado por Lamas, 2015). Hace tres quinquenios, Pizarro (1985) refería el rendimiento académico como una medida de las capacidades respondientes o indicativas que manifiestan, en forma estimativa, lo que una persona ha aprendido como consecuencia de un proceso de instrucción o formación (Citado por Lamas, 2015).

Las dinámicas y metodologías implementadas por los docentes en el aula juegan un papel fundamental en el optimo rendimiento escolar del alumno, Cruz et al. (2004) mencionan que "para que ello sea posible es importante destacar, que, dentro del proceso de enseñanza, los docentes deben implementar diferentes estrategias para la atención de las diferencias individuales de los estudiantes" (p. 9).

En el rendimiento académico intervienen factores como el nivel intelectual, la personalidad, la motivación, las aptitudes, los intereses, los hábitos de estudio, la autoestima o la relación profesor-alumno; cuando se produce un desfase entre el rendimiento académico y el rendimiento que se espera del alumno, se habla de rendimiento discrepante; un rendimiento académico insatisfactorio es aquel que se sitúa por debajo del rendimiento esperado. En ocasiones puede estar relacionado con los métodos didácticos (Citado por Lamas, 2015). 
Por otra parte, la implicación de la familia en la tarea educativa comprende no solo una participación activa de los padres en los proyectos educativos de la escuela, sino además como mediadores del aprendizaje (Citado por Jadue, Gladys,1999).

Burgos (2004) expresa que "la familia, en cualquier sociedad, es el lugar de socialización primaria, es decir, el instrumento que permite al sujeto aprender y adquirir los conocimientos y capacidades para entrar en relación con los otros" (p. 35).

Este compromiso implica compartir la información, asistir como voluntario a la clase, ayudar a los hijos en la casa. Los padres, como primeros profesores de los niños, juegan un papel muy significativo en el proceso de aprendizaje y de socialización.

La institución escolar coloca una serie de demandas sobre los alumnos y, a través de ellos, sobre el núcleo familiar. Los padres se prueban a sí mismos a través de las notas obtenidas por sus hijos, lo que provoca problemas en la conducta infantil, que desembocan en una triangulación de los conflictos intrafamiliares y de los padres con la escuela (Citado por Jadue, Gladys,1999).

El niño enfrenta constantemente situaciones académicas, cuyas exigencias debe comparar con sus propios medios. Cuando su evaluación de las demandas de la escuela lo llevan a concluir que son más de lo que él puede rendir, afronta una situación de peligro, de humillación, a veces suficiente para justificar el rechazo a la escuela. En general, la humillación originada por pequeños fracasos escolares es transitoria. Sin embargo, algunos niños sienten sus fracasos más que otros. Algunos llegan a la escuela después de haber sufrido en el hogar mayores fracasos que sus compañeros (Citado por Jadue, Gladys, 1999).

El apoyo de los padres hacia sus hijos aparece determinado por una valoración de los aprendizajes escolares, como un factor fundamental de desarrollo y por una valoración de la propia capacidad para apoyar este proceso, independientemente del nivel socioeconómico y cultural al que pertenecen, pero también se asocian a las características de los padres y de los hijos el contexto familiar y las actitudes de los profesores hacia los padres y hacia los niños. (Citado por Jadue, Gladys,1999).

El estudio del rendimiento académico de los estudiantes es, por su relevancia y complejidad, uno de los temas de mayor controversia en la investigación educativa y se le ha dedicado especial atención en las últimas décadas.

Diversos autores coinciden al sostener que el rendimiento académico es el resultado del aprendizaje suscitado por la actividad didáctica del profesor y 
producido en el alumno. Para Martínez-Otero (2007), desde un enfoque humanista, el rendimiento académico es "el producto que da el alumnado en los centros de enseñanza y que habitualmente se expresa a través de las calificaciones escolares" (p.34). Hace tres quinquenios, Pizarro (1985) refería el rendimiento académico como una medida de las capacidades respondientes $\mathrm{o}$ indicativas que manifiestan, en forma estimativa, lo que una persona ha aprendido como consecuencia de un proceso de instrucción o formación (Citado por Lamas, 2015).

Los sistemas educativos de gran parte de los países de Latinoamérica comparten en alguna medida los siguientes rasgos: insuficiente cobertura de la educación preescolar, elevado acceso al ciclo básico y escasa capacidad de retención, tanto en el nivel primario como en el secundario. Así, la repetición y el retraso escolar, fenómenos que con alta frecuencia anteceden a la deserción, unidos a un bajo nivel de aprendizaje de los contenidos básicos de la enseñanza, conspiran contra el aprovechamiento del potencial de los niños desde temprana edad. Sus efectos negativos se acumulan a lo largo del ciclo escolar, incidiendo de manera muy desigual en las oportunidades de bienestar, sobre todo entre los sectores más pobres (Guerrero, Cardona, Cuevas, 2013, p.656).

\section{Procedimiento}

Se eligió el I.E.D Francisco de Miranda en la localidad de Kennedy para realizar el estudio, en este se tomaron seis cursos del bloque de bachillerato, para obtener la muestra de 35 estudiantes hombres y mujeres que presentan alto número de materias perdidas en tercer y cuarto periodo, a estos se les aplicó el instrumento con una duración de aproximadamente 1 hora por estudiante. Posteriormente se sistematizó la información obtenida en una base de datos creada por los propios encuestadores.

\section{Resultados}

El 25.7\% de los estudiantes se encuentran en la edad de 14 años siendo este el porcentaje más alto. Este primer resultado pues estar directamente asociado a la adolescencia presente en el ciclo vital de cada uno de los ser humanos. Esta edad de los 14 años entra en la adolescencia media en la cual se presentan una serie de cambios particulares.

Los adolescentes tienen conciencia de los riesgos que corren, pero esto no los inhibe de presentar estas conductas debido a que atraviesan por un 
período de incremento sustancial de la inclinación hacia la búsqueda de recompensas o sensaciones, lo que aumenta en presencia de pares. Ello sería producto de la maduración más temprana del sistema cerebral socioemocional que del sistema de control cognitivo, con el resultado de que, en condiciones de excitación emocional, el primero sobrepasa la capacidad regulatoria del segundo (todavía relativamente inmaduro). (Gaete, Verónica. 2015, p.1)

En el rango de género predomina el género masculino, perteneciendo al 55.3\% de la población lo cual equivale a 21 hombres.

Por otra parte, una de las cifras más significativas es que el $42 \%$ de los estudiantes pertenecen a familiares nucleares, lo cual anula de nuestros juicios que los estudiantes que tienen bajo rendimiento escolar viven solo con uno de sus progenitores y que este factor influye en que no puedan realizar un debido acompañamiento a sus hijos en las labores escolares. En estas familias nucleares, todos los padres están vinculados laboralmente y el 75\% de las madres cuentan con vinculación laboral. Del 100\% el 50\% de los NNA no cuentan con acceso a internet en sus hogares. Podría ser este un limitante para realizar las labores académicas En este grupo particular la situación socioeconómica puede ser un factor influyente en el bajo rendimiento escolar, puesto que los jefes de familia cuentan con una situación económica estable, pero la mitad de la población no cuenta con acceso a internet en sus hogares. López (Como se citó en Serrano, 2013) La procedencia socioeconómica puede considerarse, por tanto, uno de los factores explicativos del bajo rendimiento ya que los alumnos procedentes de hogares en desventaja social y cultural están menos preparados lo que acentúa la posibilidad de obtener un rendimiento escolar por debajo del esperado.

Otro de los porcentajes significativos pertenece al $55.3 \%$ de los NNA que tienen una relación cercana con las redes sociales, esto evidenciado en el ecomapa, pues los estudiantes dedican la mayor parte de su tiempo a esta actividad.

Arab \& Díaz (Como se citó en Espinoza Guamán, E. E., Cruz Yaguachi, L. N., \& Espinoza Freire, E. E, 2018) piensa que la gravedad del mal uso de las redes sociales es ilimitada, porque no solo afecta en su comportamiento sino en los procesos cognitivos del estudiante, al enfrentarse a problemas académicos o personales. En las investigaciones realizadas por Morocco (2015), se indican que el excesivo empleo de Facebook causa en los estudiantes la desconcentración, desinterés por los estudios e inasistencias a clases, lo que repercute negativamente en el rendimiento académico. 
Dentro de los eventos vividos en la familia el $60.5 \%$ de la población manifestó hacer parte del proceso de la ruptura de la relación conyugal de sus progenitores. El divorcio es un agente de gran influencia en el rendimiento académico, el mismo que genera un bajo rendimiento, muchos opinan que posterior al divorcio la pareja se encuentra en una inestabilidad emocional que producirá incomodidad de los padres para con los hijos, esto tendrá como consecuencia que los hijos se sientan desplazados por los padres y de este desligamiento es donde se inicia un conflicto escolar, puesto que, ya no existe un interés de los padres hacia los hijos. (Ochoa, 2012, p.36)

\section{Vl. Conclusiones}

La familia juega un papel importante en el rendimiento escolar de los NNA, ya sea positiva o negativamente, esto se debe a todos los factores como que influyen en un NNA, como: estructura, capital cultural, pautas de crianza, comunicación, confianza y uno de los aspectos más importantes que se identificó en esta investigación es el acompañamiento que debe realizar uno de los miembros de la familia, que el estudiante reconozca como figura materna o paterna (tío, abuela, madre, padre, etc).

La educación de los NNA, es un trabajo reciproco, entre la institución educativa y la familia. Ya que según la investigación si existe buena relación entre estos dos sistemas, será más satisfactoria el resultado académico del estudiante.

El trabajo efectuado ha dejado líneas abiertas para posibles investigaciones futuras. Se debería llevar a cabo una investigación frente al funcionamiento del sistema académico colombiano, en cuanto a las necesidades de las nuevas generaciones. Pues bien sabemos que este sistema aun tiene raíces de actuación basadas en la revolución industrial, en este periodo se formaban individuos para atender la mentalidad de producción de masa, pues aun los estudiantes son educados para una ardua y larga vida de producción de trabajo, sin entender que este sistema ya no es apto para las nuevas generaciones, los estudiantes necesitan formarse como personas, desarrollar habilidades y capacidades que les permitan desempeñarse profesionalmente de forma eficiente y eficaz en el mercado laboral y la sociedad. Es importante que aprendan a tomar decisiones por su cuenta, a trabajar bien con los demás, crear empatía por el otro, dándole a sus acciones un fin social. Por lo que es hora de que el sistema de educación se ponga a la altura de las circunstancias y cumpla con su función. 


\section{Referencias}

Barrios, I., Y Frias, M. (2016). Factores que Influyen en el Desarrollo y Rendimiento Escolar de los Jóvenes de Bachillerato. Revista Colombiana de Psicología, volumen (25), 63-82.

Burgos, J. M. (2004). Diagnóstico sobre la familia. Madrid: Palabra.

Calvo, Isabel, Verdugo, Miguel Ángel, \& Amor, Antonio Manuel. (2016). La Participación Familiar es un Requisito Imprescindible para una Escuela Inclusiva. Revista latinoamericana de educación inclusiva, volumen (10), 99-113.

Cortéz Bohigas, M. M. (s.f.) Definición de rendimiento escolar. Recuperado el 28 de noviembre del 2012 en: www.psicopedagogia.con/definicion/rendimiento\%20escolar Cruz, G., Alpízar, A. L., Avendaño, M., Segura, M., Rodríguez, M., Torres, R., Rivera, Y. (2004). La evaluación de los aprendizajes en el contexto de la atención de las necesidades educativas de los estudiantes. San José, Costa Rica: MEP. Recuperado de http://docplayer.es/1119351- La-evaluacion-de-los-aprendizajes-en-el-contexto-de-laatencion-de-las-necesidadeseducativas-de-los-estudiantes.html

Espinoza Guamán, E. E., Cruz Yaguachi, L. N., y Espinoza Freire, E. E. (2018). Las redes sociales y rendimiento académi-co. Revista Metropolitana de Ciencias Aplicadas, volumen (1), 38-44.

Ferrel, O., Robert, F., Mendoza, V.J., Y Ballestas, F.L. (2014). Factores psicológicos en adolescentes escolarizados con bajo rendimiento académico: depresión y autoestima. Revista Encuentros, Volumen (12), 35-47.

Gaete, Verónica. (2015). Desarrollo psicosocial del adolescente. Revista chilena de pediatria, volumen (86), 436-443.

González Pienda, J .(2003). El rendimiento escolar. una análisis de las variables que lo condicionan. Revista galego-portuguesa de psicoloxía e educación, volumen (8), 247-258.

Guerrero, C., Y Cardona, A., Y Cuevas, J. (2013). Factores de riesgo asociados a bajo rendimiento académico en escolares de Bogotá. Investigaciones Andina, volumen (26), $15-108$.

Gladys, J. (1999). Hacia una mayor permanencia en el sistema escolar de los niños en riesgo de bajo rendimiento y de desercion. Revista scielo estudios pedagógicos valdivia, volumen (25), 83-90.

Gladys, J. (2003). Transformaciones familiares en chile: riesgo creciente para el desarrollo emocional, psicosocial y la educación de los hijos. Revista scielo estudios pedagógicos valdivia, volumen (29), 115-126.

Jimenez, R. (2011). Bajo rendimiento academico en los estudiantes de los grados $4^{\circ}, 5^{\circ}$ y $6^{\circ}$ de básica primaria de la sede escuela rural mixta la laguna dinde - cajibio cauca. Recuperado de: http://repositorio.ucm.edu.co:8080/jspui/bitstream/handle/10839/ 204/Robinson\%20tancredo\%20Jimenez\%20Gomez.pdf?sequence=1\&isAllowed=y Lamas, H. (2015). Sobre el rendimiento esco-lar. Propósitos y Representaciones. Revista de psicología educativa, volumen (3), 313-386.

Lozano, A., y Rioboo, A., y Blanco, J., y Fraga, H., y Enríquez, E. (2007). Contextos 
Familiares Y Rendimiento Escolar En El Alumnado De Educación Secundaria. Revista Internacional de Psicología del Desarrollo y la Educación, volumen (1), 197-217.

Muñoz (2005) Las Pautas De Crianza. Recuperado de: http://www.scielo.org.pe/scielo. php?pid=S1729-48272009000200005\&script=sci_arttext

Ochoa, E. D. (2012). El Divorcio y su influencia en el rendimiento académico en estudiantes de segundo año de bachillerato. Tesis de grado para optar a grado académico de psicóloga educativa y orientadora vocacional. Recuperado de: http:// repositorio.ug.edu.ec/bitstream/redug/5983/1/Tesis\%20de\%20Psicolog\%C3\% ADa\%20Educativa.pdfel

Rodríguez, O. J. (2015). El Rendimiento Escolar y la Intervención del Trabajo Social. Trabajo de Grado. Recuperado de: https://core.ac.uk/download/pdf/46159699.pdf Retana Bonilla, O. (s.f) Definición de rendimiento escolar. Recuperado el 28 de noviembre del 2012 en: www.psicopedagogia.con/definicion/rendimiento\%20escolar

Ruiz de Miguel, C. (2002) Factores familiares vinculados al bajo rendimiento. Revista Complutense de Educación. Volumen (12), 81-113. 
